\title{
Cryptanalysis of Knapsack Cipher Using Genetic Algorithm
}

\section{Subhi H. Hamdon Najlaa B. Al-Dabbagh Milad J. Saeed \\ College of Computer Science and Mathematics \\ University of Mosul, Iraq}

\section{Received on: 02/05/2007}

\section{ABSTRACT}

Accepted on: 26/09/2007

This research offers a new method in Cryptanalysis of knapsack cipher. It focuses on the application of genetic algorithm as a modern way in solving complex problems (problems have a huge numbers of alternate solutions in appropriate time). One of these problems is knapsack problem which is considered one of the known problems in operation researches. Cryptanalysis is done by using a new algorithm that is different from known knapsack breaking algorithm. Genetic algorithm has recently been successfully applied to the cryptanalysis of ciphers, among them Substitution ciphers and Transposition ciphers. This research deals with another type of ciphers called Public-key ciphers, that are high secure ciphers because they are based on NP-Complete problems.

Keywords: Cryptography, Cryptanalysis, Genetic algorithm.

$$
\begin{aligned}
& \text { تحليل شفرة نابساك باستخدام الخوارزمية الجينية }
\end{aligned}
$$

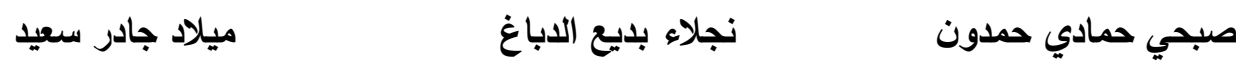

$$
\begin{aligned}
& \text { كلية علوم الحاسبات والرياضيات، جامعة الموصل بلائل } \\
& \text { تاريخ قبول البحث: 2007/09/26 } \\
& \text { تاريخ استلام البحث: 2007/05/02 }
\end{aligned}
$$

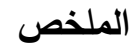

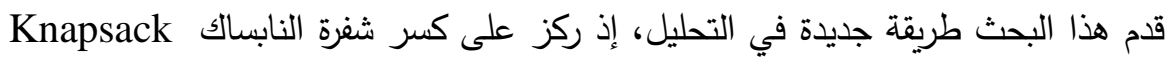

Cipher

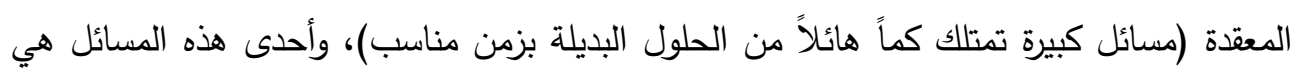

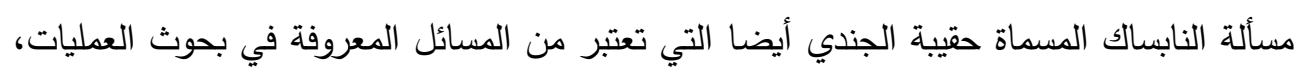

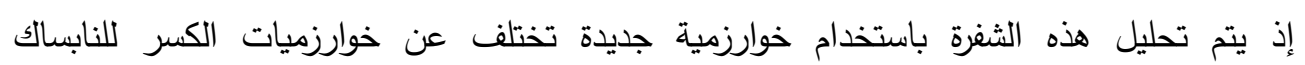

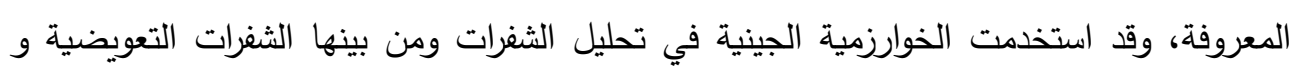

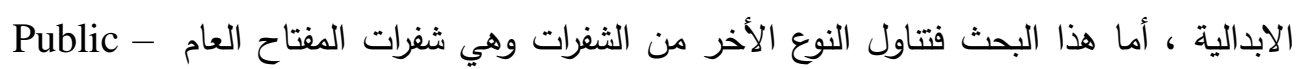

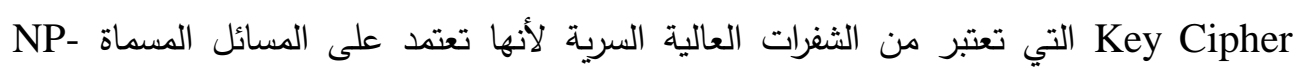
. Complete Problem الكلمات المفتاحية: التثفير، التحليل، الخوارزمية الجينية. 
علم التشفير Cryptographgy هو العلم الذي يختص بحماية المعلومات من الأشخاص

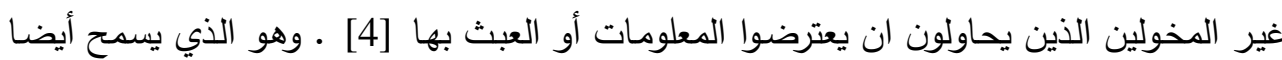

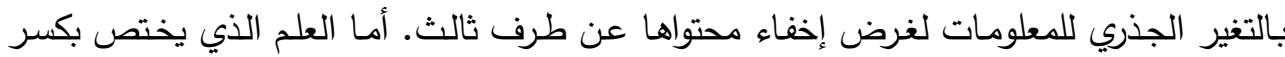
الثفرة فهو علم تحليل الثفرة Cryptanalysis. ولتحويل النص الصريح إلى نص مشفر يحتاج إلى خوارزمية ومفتاح. وهنالك نوعان من خوارزميات التثفير المعروفة [8]: - - - خوارزميات تشفير المفتاح السري التي تستخدم المفتاح نفسه في التثفير وفك الثفرة. - - خوارزميات تشفير المفتاح العـام التي تستخدم زوجا مـن المفاتيح التي تكون مترابطـة رياضياً، الأول يستخدم في التشفير والثاني يستخدم لفك الثفرة.

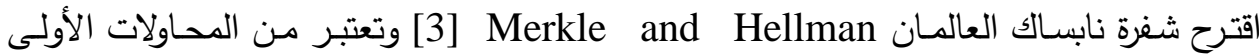
لأنظمة المفتاح العام ويطلق عليها أيضـا مسالة حقيبة الجندي وشفرة نابساك تتعلق بإيجاد حل لمسألة الجمع الجزئي Subset Sum Problem التي تعرف رياضياً كالأتي:A توجد مجموعة من الأعداد الصحيحة الموجبة ولتكن $\mathrm{A}=\{\mathrm{a} 1, \mathrm{a} 2, \mathrm{a}\}$ .an $\}$

ويوجد عدد صحيح موجب وليكن B والمطلوب هل هنالك مجموعة جزئية A من المجموعة A

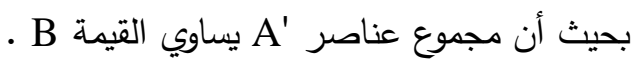
إن نابساك الاعتيادية المسماة Super increasing Knapsack من السهولة كسرها أي بمعنى

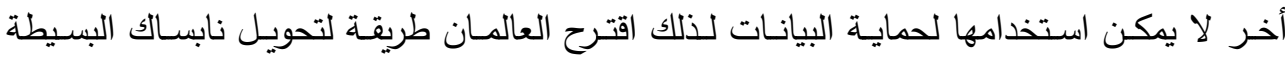
Simple Knapsack والطريقة هي كالأتي: إذ كان لديك سلسة نابساك البسيطة $\mathrm{A}=\left\{\mathrm{a}^{\prime} 1, \mathrm{a}^{\prime} 2, \mathrm{a}^{\prime} 3, \ldots \ldots \ldots . . . \mathrm{a}^{\prime} \mathrm{n}\right\}$

$$
\begin{aligned}
& \text { فأن عمليات التحويل إلى سلسلة باب المصيدة هي :- }
\end{aligned}
$$

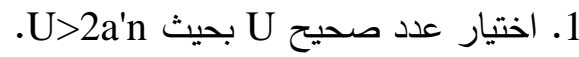

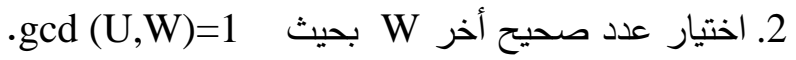

$$
\begin{aligned}
& \text { 3. إيجاد قيمة } \\
& \text { 4. تكون سلسلة باب المصيدة A= WA' mod U. }
\end{aligned}
$$


وبالرغم من أن شفرة نابساك تعتبر من مسائل NP- Complete وهي شفرة ذات سرية عالية، فأن

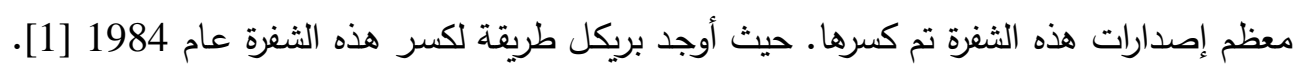

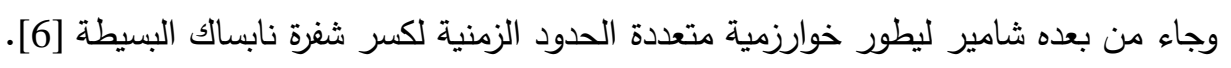

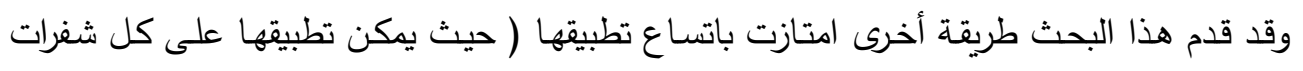
النابساك) وسهولة عملها. اعتمدت الخوارزمية الجينية لتحقيق هذا الهذف.

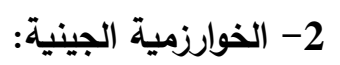

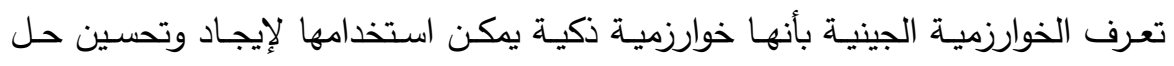

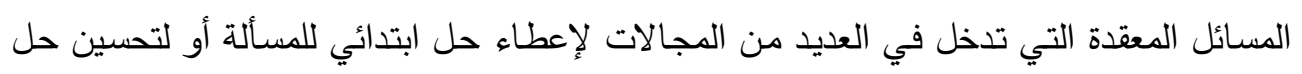

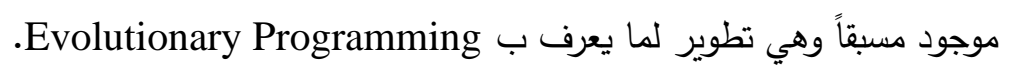

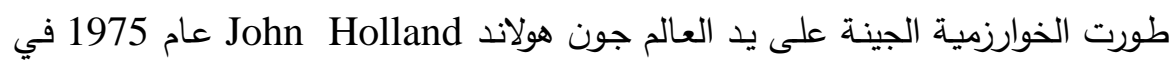

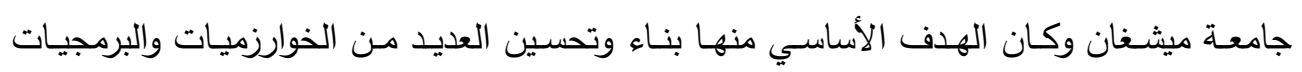

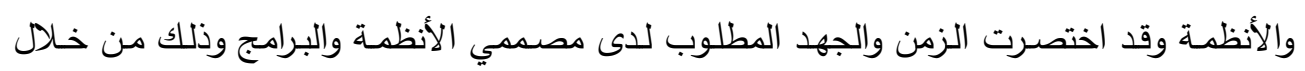

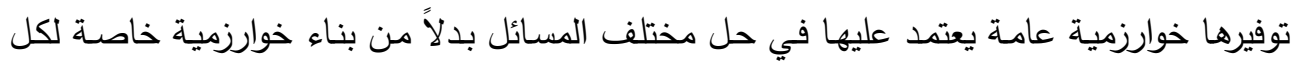

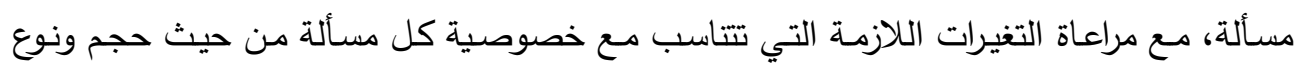
البيانات المستخدمة وطبيعة دالة الهدف وقيود كل مسألة [2,5]

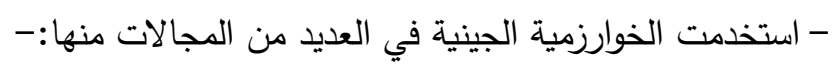

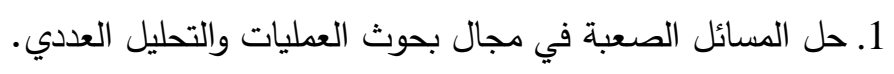

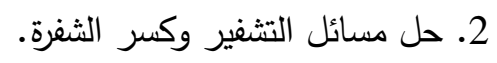

$$
\text { 4. 3. تعليم الحاسبة. }
$$

\section{• الخطوات العامة للخوارزمية الجينية:}

تتضمن الخوارزمية الجينية عدداً من الخطوات الثابتة لمختلف المسائل ولجميع التطبيقات

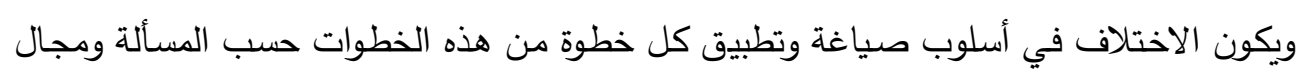

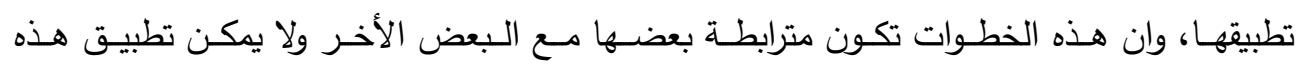

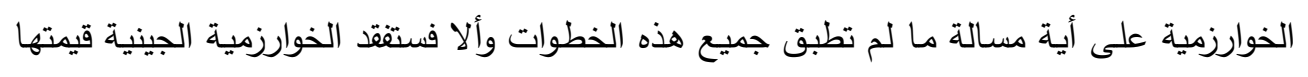
وفائدتها في إيجاد الحل وتحسينه. 
إن إنشاء الجيل الابتدائي يعد نقطة الانطلاق في تحسين حالة المسألة المراد تطبيقها. وان جميع المصادر المتوافرة تبين أن عملية بناء الجيل الابتدائي تتم بطريقة عشوائية.

- دالة الهُف وقيمة اللياقة واحتمالية المساهمة •

تحسب دالة الهدف الخاصة لمسألة ما لكل مقطع من مقاطع الجيل.

Fitness Value مدى اللياقة:

تحسب قيمة مدى اللياقة لكل مقطع من مقاطع الجيل اعتماداً على قيمة دالة الهدف لذلك المقطع. وتحدد قيمة مدى اللياقة مقدار جودة المقطع قياساً بباقي مقاطع الجيل.

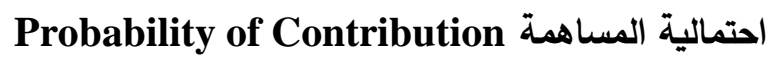

تجمع درجات اللياقة لجميع مقاطع الجيل لكي تستخدم في إيجاد احتمالية مساهمة كل

مقطع في تكوين الجيل اللاحق والتي تحسب بقسمة درجة اللياقة لكل مقطع على قيمـة مجموع درجات اللياقة.

• بعد حساب دالة الهدف ومدى اللياقة واحتمالية المساهمة لجميع مقاطع الجيل الابتدائي تبدأ عملية تكوين الجيل الجديد بتطبيق عدد من العمليات على المقاطع المنتخبة من الجيل الحالي وتتمثل هذه العمليات بالدوال الثلاث آلاتية:-

- اختيار الآباء Parents Selection -

هذه العملية تحدد أي مقطع في هذا الجيل سوف يساهم في توليد الجيل اللاحق وهناك

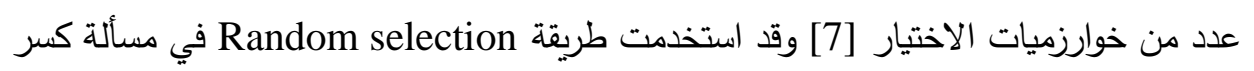
شفرة Knapsack.

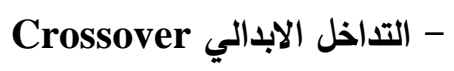
يتكون مقطع جديد ليمثل احد مقاطع الجيل اللاحق من مقطعين من مقاطع الآباء المنتخبة من مقاطع الجيل الحالي باستخدام إحدى طرائق التداخل المعروفة [2].

Mutation (التغير ضمن المقطع (الطفرة) -

تتمثل هذه العملية بأجراء تغيير أو تبديل بين قيم محددة ضمن المقطع نفسه لتكوين وإدخال

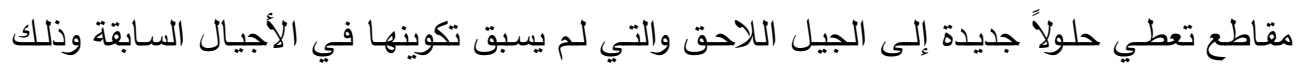
بهدف توسيع حيز الحلول المكنة والتثثيل بتكوين اكبر عدد من المقاطع المختلفة ضدن الجيل.

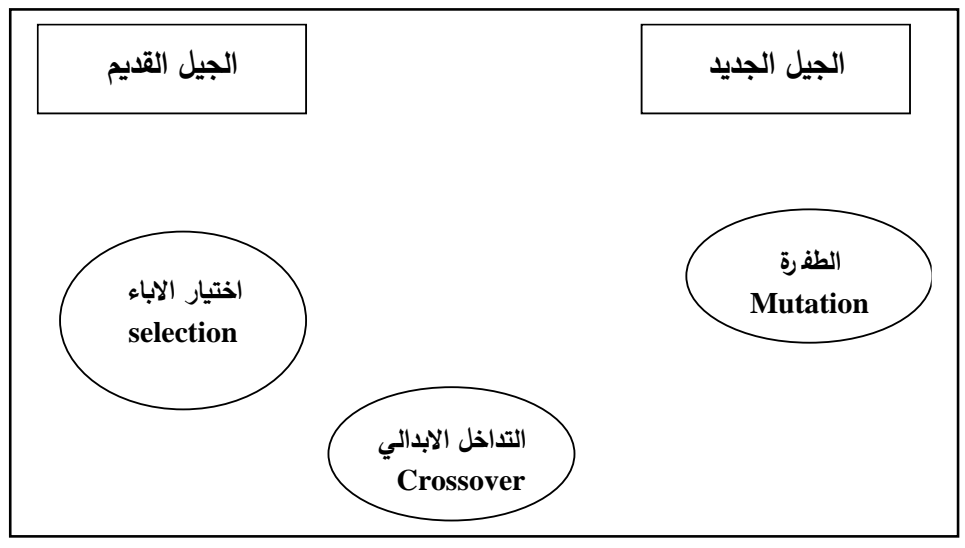




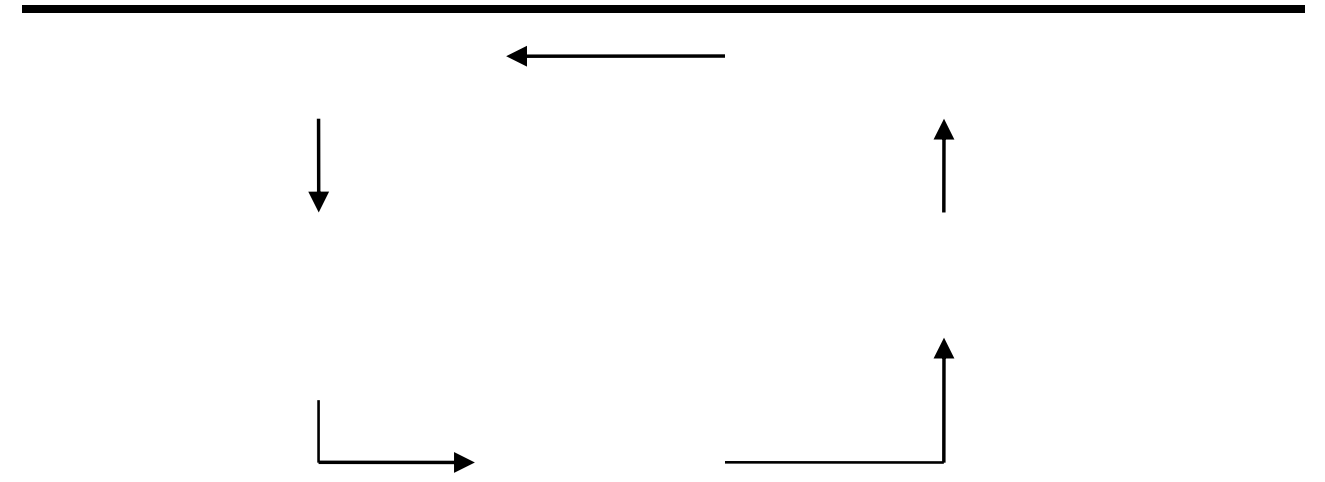

\title{
الثكل (1) يوضح دورة حياة الخوارزمية الجينية
}

\begin{abstract}
• • معيار توقف الخوارزمية الجينية:
يستمر تكوين الأجيال المتعاقبة بهدف تحسين الحل أي يجعله أكثر اقتراباً من الحل الامثل إلى

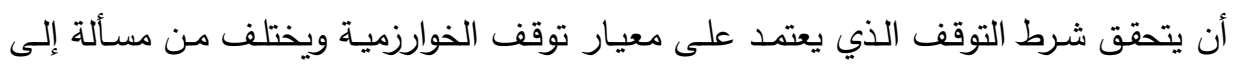
أخرى، ومن أهم معايير التوقف وأكثرها استخداماً: • التحديد المسبق لعدد الأجيال المتكونة.

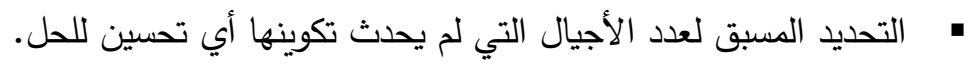

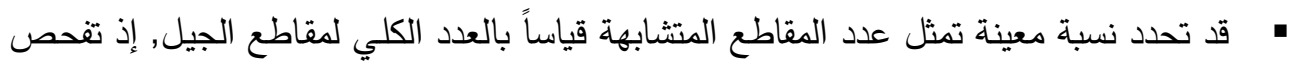

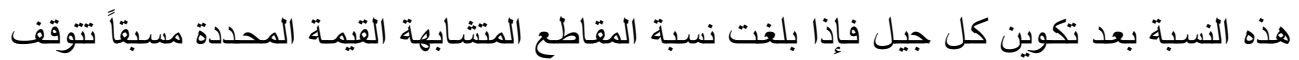
الخوارزمية.
\end{abstract}

3- 3 ت تحليل شفرة نابساك:

اعتمدت طريقة التحليل على ثلاث خطوات رئيسية:

أ- التمثيل ودالة الهذف 
الهيكل التمثيلي لمشكلة النابساك سهل التوليد لان المسألة تقترح صيغة طبيعية الثكل وهو

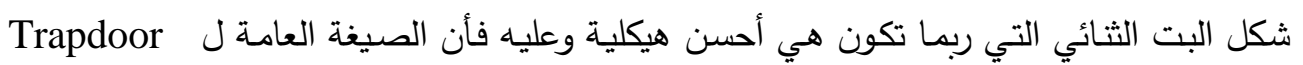
KnapSack

إضافة القيم الموجودة في المو اقع 1, 3, 5, 7

\begin{tabular}{|l|l|l|l|l|l|l|l|}
\hline 1 & 0 & 1 & 0 & 1 & 0 & 1 & 0 \\
\hline
\end{tabular}

إضافة القيم الموجودة في المو اقع 2, 3, 5, 6

\begin{tabular}{|l|l|l|l|l|l|l|l|}
\hline 0 & 1 & 1 & 0 & 1 & 1 & 0 & 0 \\
\hline
\end{tabular}

عندما يتم التمثيل للخوارزمية الجينية من الضروري إضافة دالة الهدف التي تستخدم لتحديد أفضل

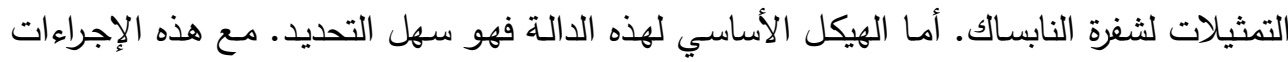
العامة يوجد مدى واسع للمتغيرات المحتملة، في هذا البحث فأن دالة الهدف الحقيقية يجب أن يكون لها ثلاث خصائص:

• مدى الدالة يجب أن يكون بين الصفر والواحد، إذ أن الواحد يمثل التطابق التام مع مجموع الهدف للنابساك.

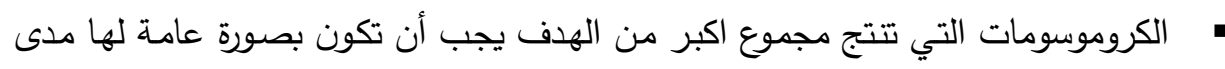
اللياقة اقل من الكروموسومات التي تتتج مجموع اقل من الهدف، بهذه الطريقة فان الحلول غير العملية هي التي تتتج مجموع أكثر من الهدف المطلوب بينما الحلول العملية لها فرصة اكبر من أن تتماشى مع الخوارزمية. يجب أن تكون هناك صعوبة في إنتاج قيمة لياقة عالية ، وان الاختلافات الصغيرة الكئه بين

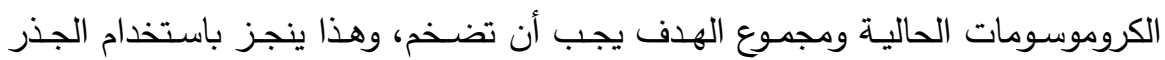
ألتربيعي في حساب الدالة المختارة. إن دالة الهدف الحقيقة التي تستعمل في هذا البحث تستخدم لتحديد ما يأتي: 1. حساب أعلى اختلاف قد يحدث بين الكروموسومات ومجموع الهدف MaxDiff = max (Target, Full sum - Target ) حيث Fullsum هو مجموع كل مكونات النابساك.

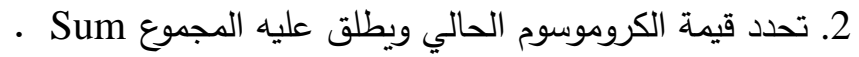
3. إذا كان المجموع اقل أو يساوي الهدف فاللياقة للكروموسوم تحسب الحبه كما يأتي: 
Fit $=1$-sqrt $(\mid$ sum - target $\mid /$ Target $)$

$$
\text { 4. }
$$

$$
\text { ب-عملية التزاوج }
$$

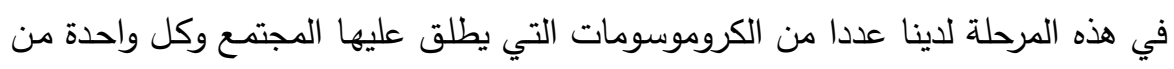

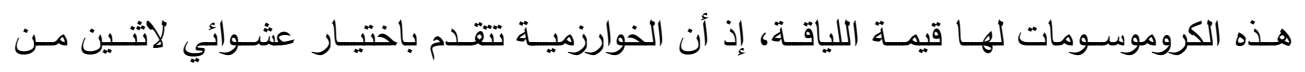

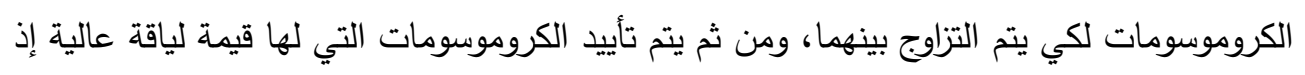

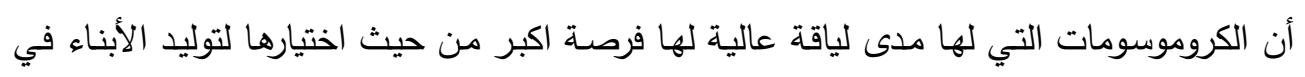

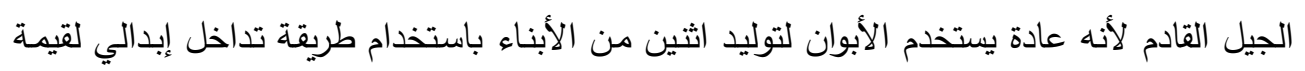
واحدة.

ج- عملية الطفرة

هذه المرحلة تساهم في توليد أبناء جدد لكي تجنب الخوارزميـة الوقوع في نقطة الحل المثلى المحلية Local Optimal وقد تم استخدام المتمم أن تتم مقارنة قيمة الاحتمالية المختارة عشوائياً مع القيمة المدخلة من التتفيذ.

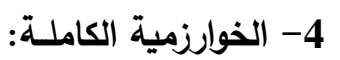

هذه المعالجات تتعاون لخلق خوارزمية جينية متكاملة، تبدأ بنص مشفر باستخدام شفرة

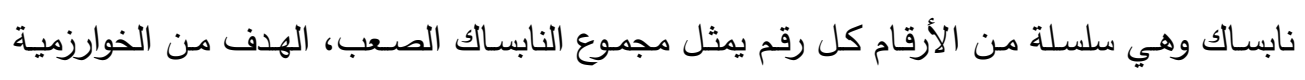

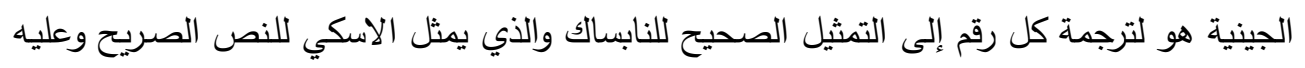
فان النقاط التالية تُفذ لكل رقم مشفر وهي كالأتي:

1. توليد مجتمع عشوائي للكروموسومات ( سلسلة ثنائية من الاصفار والواحدات).

$$
\text { 3. 2. حساب مدى اللياقة لكل كروموسوم في المجتمع. }
$$

4. تطبيق عملية التزاوج على الآباء المختارة.

5. تطبيق عملية الطفرة على الأبناء.

إن هذه العملية سوف تتوقف بعد عدد ثابت من الأجيال وأفضل كروموسوم هو الذي سوف يستخدم الأناء لفك شفرة النص المشفر ( العدد). 5- - 5 النتائج والتطبيق العملي: 
تمت برمجة خوارزمية التحليل باستخدام لغة Delphi البرمجية الواجهة الرئيسية للبرنامج موضحة بالثكل (2) ويمكن توضيح استخدام الخوارزمية بالمثال العملي الأتي:

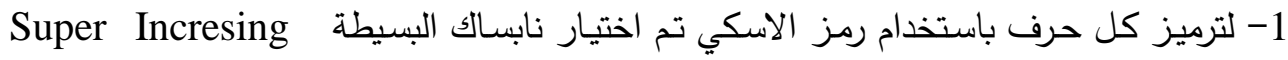
فهي Wnapsack

$$
\mathrm{W}=13, \mathrm{~N}=672
$$

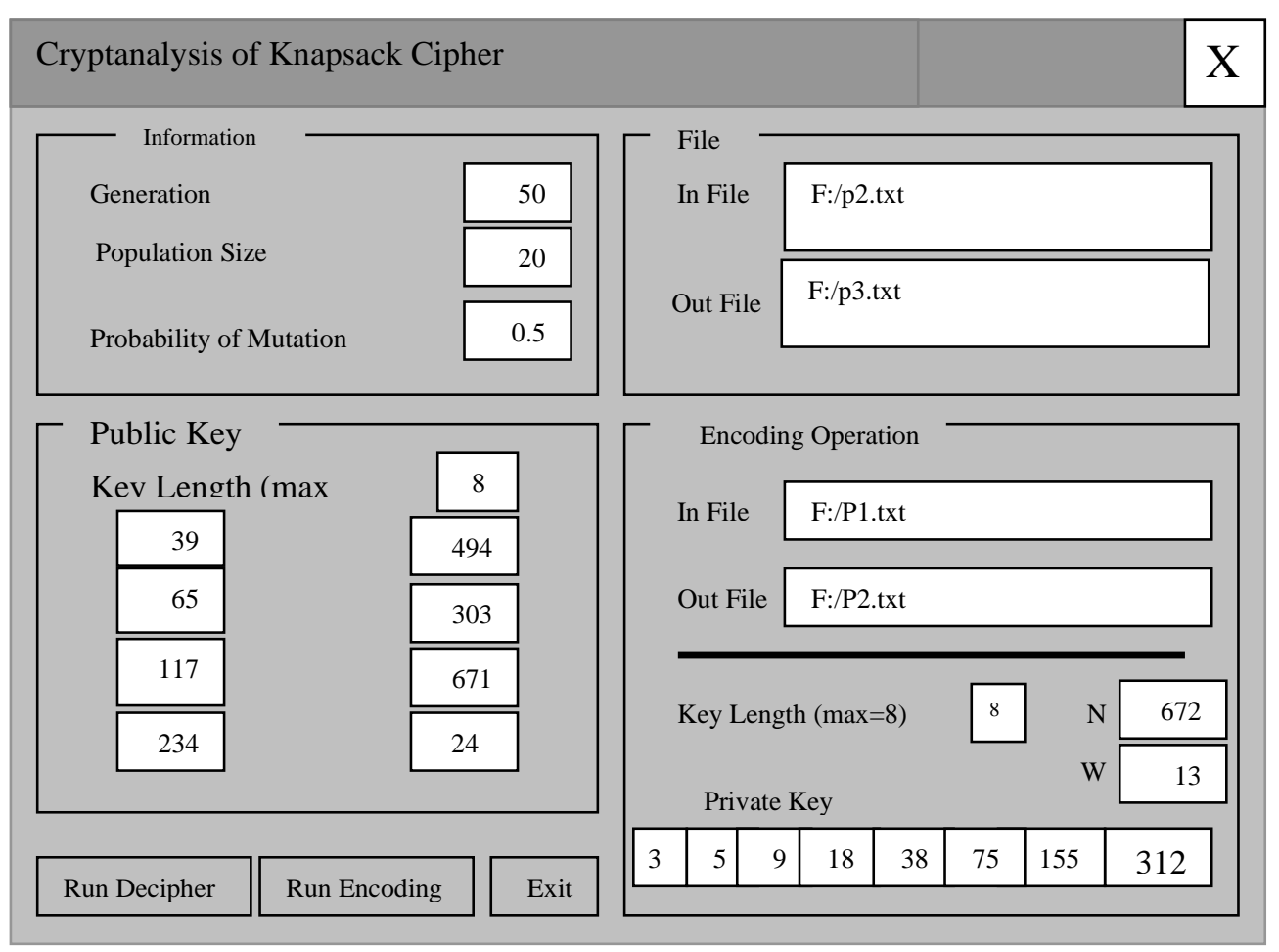

الثكل (2) واجهة البرنامج

2- تم تكوين نابساك الصعب Hard Knapsack وكانت

$\{39,65,117,234,494,303,671,24\}$

$$
\text { 3- اختير النص الواضح التالي ليتم تشفيره }
$$




$$
\text { 4- النص المشفر كان:- }
$$

$\{710,1087,1126,1165,827,1230,710,1282,944,1087,892,1269,1438,1269,12$ $82,827,1061,944,1279,710,1165,1230,1126,892,1230,710,1061,1282,905,7$ $10,1282,710,775,1282,1269,710,1269,710,1087,944,1087,1282,827,1230,8$ $27,788,944,710,1230,1438,736,827,1282,1386,827,827,1089,710,1231,126$ $9,827,1230,1126,853,710,775,1126,1061,1165,1231,1282,827,1230,710,10$ $87,788,1282,905,827,775,1126,1061,1165,1231,1282,827,1230,905,710,12$ $30,788,1386,710,1230,827\}$

5- أما النتائج فموضحة بالجدول (1):-

\begin{tabular}{|c|c|c|c|c|}
\hline Key & Sum & Fitness & Char & Best Gen \\
\hline 10000010 & 710 & 1.0000 & A & 35 \\
\hline 01110010 & 1087 & 1.0000 & N & 8 \\
\hline 1110010 & 1126 & 1.0000 & O & 8 \\
\hline 00001010 & 1165 & 1.0000 & P & 10 \\
\hline 10100010 & 827 & 1.0000 & E & 13 \\
\hline 01001010 & 1230 & 1.0000 & R & 24 \\
\hline 10000010 & 710 & 1.0000 & A & 5 \\
\hline 00101010 & 1282 & 1.0000 & T & 2 \\
\hline 10010010 & 944 & 1.0000 & I & 6 \\
\hline 01110010 & 1087 & 1.0000 & N & 9 \\
\hline 11100010 & 892 & 1.0000 & G & 1 \\
\hline 11001010 & 1269 & 1.0000 & S & 29 \\
\hline 10011010 & 1438 & 1.0000 & Y & 21 \\
\hline 11001010 & 1269 & 1.0000 & S & 46 \\
\hline 00101010 & 1282 & 1.0000 & T & 3 \\
\hline 10100010 & 827 & 1.0000 & E & 15 \\
\hline 10110010 & 1061 & 1.0000 & M & 6 \\
\hline 10010010 & 944 & 1.0000 & I & 3 \\
\hline 11001010 & 1269 & 1.0000 & S & 17 \\
\hline 10000010 & 710 & 1.0000 & A & 15 \\
\hline 00001010 & 1165 & 1.0000 & P & 16 \\
\hline 01001010 & 1230 & 1.0000 & R & 11 \\
\hline 11110010 & 1126 & 1.0000 & O & 4 \\
\hline 11100010 & 892 & 1.0000 & G & 1 \\
\hline 01001010 & 1230 & 1.0000 & R & 3 \\
\hline 10000010 & 710 & 1.0000 & A & 11 \\
\hline 10110010 & 1061 & 1.0000 & M & 1 \\
\hline 00101010 & 1282 & 1.0000 & T & 2 \\
\hline 00010010 & 905 & 1.0000 & H & 1 \\
\hline 10000010 & 710 & 1.0000 & A & 8 \\
\hline 00101010 & 1282 & 1.0000 & T & 1 \\
\hline 10000010 & 710 & 1.0000 & A & 3 \\
\hline 11000010 & 775 & 1.0000 & C & 35 \\
\hline 00101010 & 1282 & 1.0000 & T & 3 \\
\hline 11001010 & 1269 & 1.0000 & S & 17 \\
\hline 10000010 & 710 & 1.0000 & A & 15 \\
\hline 11001010 & 1269 & 1.0000 & S & 12 \\
\hline 10000010 & 710 & 1.0000 & A & 24 \\
\hline 01110010 & 1087 & 1.0000 & N & 27 \\
\hline & & & & \\
\hline
\end{tabular}




\begin{tabular}{|c|c|c|c|c|}
\hline 10010010 & 944 & 1.0000 & $\mathrm{I}$ & 4 \\
\hline 01110010 & 1087 & 1.0000 & $\mathrm{~N}$ & 4 \\
\hline 00101010 & 1282 & 1.0000 & $\mathrm{~T}$ & 10 \\
\hline 10100010 & 827 & 1.0000 & $\mathrm{E}$ & 26 \\
\hline 01001010 & 1230 & 1.0000 & $\mathrm{R}$ & 23 \\
\hline 10100010 & 827 & 1.0000 & $\mathrm{E}$ & 6 \\
\hline 00100010 & 788 & 1.0000 & $\mathrm{D}$ & 1 \\
\hline 10010010 & 944 & 1.0000 & $\mathrm{I}$ & 17 \\
\hline 10000010 & 710 & 1.0000 & $\mathrm{~A}$ & 17 \\
\hline 01001010 & 1230 & 1.0000 & $\mathrm{R}$ & 33 \\
\hline 10011010 & 1438 & 1.0000 & $\mathrm{Y}$ & 1 \\
\hline 01000010 & 736 & 1.0000 & B & 6 \\
\hline 10100010 & 827 & 1.0000 & $\mathrm{E}$ & 3 \\
\hline 00101010 & 1282 & 1.0000 & $\mathrm{~T}$ & 1 \\
\hline 11101010 & 1386 & 1.0000 & $\mathrm{~W}$ & 12 \\
\hline 10100010 & 827 & 1.0000 & $\mathrm{E}$ & 9 \\
\hline 10100010 & 827 & 1.0000 & $\mathrm{E}$ & 2 \\
\hline 01110010 & 1087 & 1.0000 & $\mathrm{~N}$ & 5 \\
\hline 10000010 & 710 & 1.0000 & $\mathrm{~A}$ & 16 \\
\hline 10101010 & 1321 & 1.0000 & $\mathrm{U}$ & 12 \\
\hline 11001010 & 1269 & 1.0000 & $\mathrm{~S}$ & 6 \\
\hline 10100010 & 827 & 1.0000 & $\mathrm{E}$ & 25 \\
\hline 01001010 & 1230 & 1.0000 & $\mathrm{R}$ & 49 \\
\hline 11110010 & 1126 & 1.0000 & $\mathrm{O}$ & 1 \\
\hline 01100010 & 853 & 1.0000 & $\mathrm{~F}$ & 16 \\
\hline 10000010 & 710 & 1.0000 & A & 51 \\
\hline 11000010 & 775 & 1.0000 & $\mathrm{C}$ & 20 \\
\hline 11110010 & 1126 & 1.0000 & $\mathrm{O}$ & 55 \\
\hline 10110010 & 1061 & 1.0000 & $\mathrm{M}$ & 12 \\
\hline 00001010 & 1165 & 1.0000 & $\mathrm{P}$ & 1 \\
\hline 10101010 & 1231 & 1.0000 & $\mathrm{U}$ & 3 \\
\hline 00101010 & 1282 & 1.0000 & $\mathrm{~T}$ & 6 \\
\hline 10100010 & 827 & 1.0000 & $\mathrm{E}$ & 15 \\
\hline 01001010 & 1230 & 1.0000 & $\mathrm{R}$ & 4 \\
\hline 10000010 & 710 & 1.0000 & $\mathrm{~A}$ & 44 \\
\hline 01110010 & 1087 & 1.0000 & $\mathrm{~N}$ & 2 \\
\hline 00100010 & 788 & 1.0000 & $\mathrm{D}$ & 1 \\
\hline 00101010 & 1282 & 1.0000 & $\mathrm{~T}$ & 7 \\
\hline 00010010 & 905 & 1.0000 & $\mathrm{H}$ & 1 \\
\hline 10100010 & 827 & 1.0000 & $E$ & 1 \\
\hline 11000010 & 775 & 1.0000 & $\mathrm{C}$ & 17 \\
\hline 11110010 & 1126 & 1.0000 & $\mathrm{O}$ & 16 \\
\hline 10110010 & 1061 & 1.0000 & $\mathrm{M}$ & 2 \\
\hline 00001010 & 1165 & 1.0000 & $\mathrm{P}$ & 34 \\
\hline 10101010 & 1321 & 1.0000 & $\mathrm{U}$ & 6 \\
\hline 00101010 & 1282 & 1.0000 & $\mathrm{~T}$ & 3 \\
\hline 10100010 & 827 & 1.0000 & $\mathrm{E}$ & 15 \\
\hline 01001010 & 1230 & 1.0000 & $\mathrm{R}$ & 17 \\
\hline 00010010 & 905 & 1.0000 & $\mathrm{H}$ & 9 \\
\hline 10000010 & 710 & 1.0000 & $\mathrm{~A}$ & 20 \\
\hline 01001010 & 1230 & 1.0000 & $\mathrm{R}$ & 29 \\
\hline 00100010 & 788 & 1.0000 & $\mathrm{D}$ & 6 \\
\hline
\end{tabular}




\begin{tabular}{|c|c|c|c|c|}
\hline 11101010 & 1386 & 1.0000 & W & 8 \\
\hline 10000010 & 710 & 1.0000 & A & 8 \\
\hline 01001010 & 1230 & 1.0000 & R & 13 \\
\hline 10100010 & 827 & 1.0000 & E & 3 \\
\hline
\end{tabular}

الجدول (1) النتائج

$$
\text { 6 - 6 الاستنتاجات و التوصيات : }
$$

استخدمت الخوارزمية الجينية في تحليل شفرة نابساك التي تعتبر أداة جديدة وقوية للتحليل

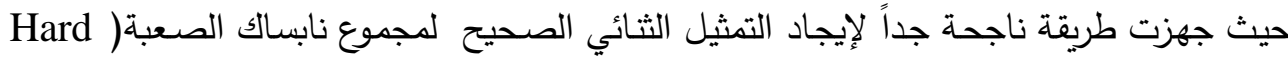

Knapsack الذي سوف تطبق عليه الخوارزمية يعتمد على حجم الذاكرة المتوافرة للإلة المستخدمة.

هنالك عدة مجالات مفتوحة للأعمال المستقبلية، فعلى سبيل المثال ممكن تحسين كفاءة الخوارزمية إذا تمت معرفة بعض حروف النص ومن جهة أخرى يمكن تطبيق الخوارزمية الجينية لكسر شفرات أكثر تعقيداً.

المصسادر

[1] Brickell, E. (1984) "Solving low Density Knapsacks", Advances in Cryptolog3/: proceedings of CRYPTO 88. NewYork: Plenum Press. Pp.25-37.

[2] Delman B. (2004) "Genetic Algorithms in Cryptography", A thesis Submitted in partial Fulfillment of the Requirements of the Degree of Master of Science in Computer Engineering, Rochester, New York, July. 
[3] Fed piper (1982) "Cipher System The Protection of Communications"

[4] Gurpreet Dhillon. (2007) Principles of Information Systems Security, John Wiley \& Sons.

[5] Rawlins, G. (1991) Foundations of Genetic Algorithms ,los Altos CA: Morgan Kaufmann Publishers.

[6] Shamir, A. (1984) "A polynomial - Time Algorithm for Breaking the Basic Merkle- Hellman Cryptosystem. IEEE Transactions on Information Theory. IT30:PP.699-704.

[7] Spillman R.; Janssen M.; Nelson B. and Kepner M. (1993) "Use of A Genetic Algorithm in analysis of simple Substitution Ciphers",Department of Computer Science Pactific Lutheran , University Tacoma WA 98447 USA.

[8] Wiley,J. \&Sons (1996) “Applied Cryptography”. 